\title{
Conformal invariance of chiral edge theories
}

\author{
N. Read \\ Department of Physics, Yale University, P.O. Box 208120, New Haven, CT 06520-8120, USA
}

(Dated: March 6, 2009)

\begin{abstract}
The low-energy effective quantum field theory of the edge excitations of a fully-gapped bulk topological phase corresponding to a local Hamiltonian must be local and unitary. Here it is shown that whenever all the edge excitations propagate in the same direction with the same velocity, it is a conformal field theory. In particular, this is the case in the quantum Hall effect for model "special Hamiltonians", for which the ground state, quasihole, and edge excitations can be found exactly as zero-energy eigenstates, provided the spectrum in the interior of the system is fully gapped. In addition, other conserved quantities in the bulk, such as particle number and spin, lead to affine Lie algebra symmetries in the edge theory. Applying the arguments to some trial wavefunctions related to non-unitary conformal field theories, it is argued that the Gaffnian state and an infinite number of others cannot describe a gapped topological phase because the numbers of edge excitations do not match any unitary conformal field theory.
\end{abstract}

\section{INTRODUCTION}

There has been renewed interest recently in fractional quantum Hall systems, especially non-Abelian ones [1], in connection with topological quantum computing. Such systems are examples of topological phases in two space dimensions, that is systems with a gap for all bulk excitations (i.e. those far from any boundary), and no apparent broken symmetries or conventional (i.e. local) order parameter. Topological phases can be distinguished by the quantum numbers and statistics (defined by adiabatic exchange) of their point-like excitations [1]. Topological phases in general may possess gapless excitations at an edge, that is the boundary with vacuum outside, or also at an interface between regions of the system in distinct topological phases. When there are gapless edge excitations, they can be described by some effective lowenergy theory. This theory has generally been expected to be a conformal field theory (CFT). However detailed arguments for this appear to be lacking in the condensed matter situation, in which for example Lorentz invariance cannot be taken for granted.

In this note we discuss conformal invariance properties of an effective field theory of the edge, with particular reference to quantum Hall (QH) systems. We show that when the bulk (interior) of the system is fully gapped and all edge excitations propagate in the same direction with the same velocity, the edge theory is a local unitary conformal field theory. These conditions are sometimes met by the "special Hamiltonians", which exist for some of the bulk topological QH phases; these have zero-energy eigenfunctions that can be found explicitly, and which are given by correlation functions in some CFT which is also the natural candidate for the corresponding effective edge theory [1]. At the end we apply these remarks to disqualify some recently-proposed trial wavefunctions from describing a topological phase.

\section{SPECIAL HAMILTONIANS}

First we address the special Hamiltonians just mentioned. Many examples of these are now known, see for instance [2, 3, 4, [5, [6] (in general we can allow the particles to have internal "spin" quantum numbers, and corresponding symmetries of the Hamiltonian may act on these indices). In each case, there is a short-range interaction for particles in the lowest Landau level (LLL). For a QH system in the infinite plane, the LLL states for a single particle in the symmetric gauge are spanned by the functions $z^{m} e^{-|z|^{2} / 4}$ with $m=0,1, \ldots$ (we have set the magnetic length to 1 ). As the single-particle states in the LLL are degenerate, the many-particle Hamiltonian $H_{0}$ can be taken to be solely the interaction, acting in the space of many-particle states in the LLL. Then, for suitable $H_{0}$, the full space of zero-energy eigenfunctions in the infinite plane can be obtained (possibly only after some effort). The simplest of all examples is

$$
H_{0}=V_{0} \sum_{i<j} \delta^{2}\left(z_{i}-z_{j}\right)
$$

projected to the LLL [2], and acting in the space of symmetrized LLL wavefunctions, describing bosons; $V_{0}>0$ is a constant. In some cases the special Hamiltonian contains many-body (say, up to some fixed $k$ independent of the particle number $N$ ) local interactions. This Hamiltonian is not intended to be directly physical, but serves as a paradigm and an existence proof for a particular phase of matter. The angular momentum $M$ is defined in two dimensions for the LLL as $M=\sum_{i} z_{i} \partial / \partial z_{i}$ (where the derivatives act only on the analytic part of the wavefunction), and always commutes with the Hamiltonian. As in the case of the Hamiltonian, it can be viewed as an integral of a local operator in the two-dimensional space. We will consider only the cases in which there is a unique zero-energy eigenstate of lowest angular momentum $M=M_{0}$, which corresponds to the ground state of the phase in question, in the form of a circular drop (disk) of radius $R$ as in Laughlin's original work [7]. (In the example of $H_{0}$ above, this ground state is the Laughlin state 
of exponent 2 for bosons at filling factor $\nu=1 / 2$.) "Lowlying" excitations above this ground state are defined as zero-energy eigenstates with a small increase in $M$; more formally, that is $\Delta M=M-M_{0}$ of order 1 as the particle number $N \rightarrow \infty$. For these it is useful to add to the special Hamiltonian $H_{0}$ the term $\omega M$ for $\omega>0$. Then as $M$ commutes with $H=H_{0}+\omega M$, the zero energy eigenstates are unchanged, and span subspaces, with one subspace for each value of $M$. Then the energy of the eigenstates increases with $M$, but is the same for each $M$.

We will introduce one further crucial assumption about the spectrum of $H_{0}$ : in the bulk of the system (as $N \rightarrow \infty$ ), there is a gap that converges to a nonzero constant in the thermodynamic limit $N \rightarrow \infty$. This is not the case for some known special Hamiltonians, but based on numerical work does appear to be the case for others. When this requirement holds, one can argue that the lowlying zero-energy eigenstates of $H_{0}$ represent excitations located at the edge of the circular drop describing the system. (Note that other states associated with particles far outside the drop will have energies approaching zero at high $M$.) For the Hamiltonian $H=H_{0}+\omega M$, these possess energies above that of the ground state, but only by amounts of order $\omega \Delta M$, so $\omega>0$ is an angular velocity. Then the velocity of propagation of the edge excitations is $v=\omega R$. There are also quasihole excitations of the system, which are zero-energy eigenstates with angular momentum $\Delta M$ of order 1 as $N \rightarrow \infty$.

\section{EFFECTIVE FIELD THEORY}

Now let us consider this system from the point of view of an effective quantum field theory for the edge. This field theory has one space and one time dimension; the space dimension is periodic, of course. For the limit of large systems, the circumference of the edge becomes arbitrarily large, and we can ignore this periodic boundary condition, and consider properties of the theory locally on the edge. The quantum field theory is unitary. A unitary field theory may be defined as one that has both a positive-definite inner product on its space of states (making this space a true Hilbert space), and a Hamiltonian that is self-adjoint with respect to this inner product. Both properties are required in all conventional quantum mechanical systems, and for the effective theory of our edge both continue to hold when we integrate out other higher energy states (non-zero-energy states of $\left.H_{0}\right)$ to derive the effective Hamiltonian. Due to the locality of the underlying Hamiltonian $H$, and the gap in the bulk excitation spectrum, the effective Hamiltonian will be an integral along the edge of a local operator $T /(2 \pi)$ (the factor $2 \pi$ here and below is conventional in CFT); thus the effective theory is also local. Local observables of the original system will also remain local when the corresponding operators in the effective edge theory are derived. For a local quantum field theory, we may refine our definition of unitarity by replacing it with the more stringent condition that the Hamiltonian density, or better the stress-energy-momentum tensor, is self-adjoint. The stress-energy-momentum tensor $T_{\mu \nu}$ of a field theory, which will be called the stress tensor for short, is the Noether current operator associated with translational invariance in space and time; here $\mu, \nu=x, t$, where $x$ is a coordinate along the edge. In general, the Hamiltonian density is the time-time component $T_{00} /(2 \pi)$ of the stress tensor.

For the edge theory, all excitations are chiral, that is they propagate along the edge, with the common velocity $v$ at sufficiently low energies. Then for any local operator $\mathcal{O}$ in the effective field theory, we have the equation of motion $\partial_{t} \mathcal{O}=-v \partial_{x} \mathcal{O}$, and so $\mathcal{O}$ is a function only of $x-v t$. Let us redefine $z=x-v t$, and choose units so that $v=1$.

For the stress tensor, one conventionally defines the light cone components with respect to the coordinates $z=x-t, \bar{z}=x+t[8,9]$. Here $z$ and $\bar{z}$ are independent real numbers. [It is sometimes useful to analytically continue by allowing $x$ and $t$ to be complex, writing $t=-i \tau$; conventional imaginary time (or Euclidean spacetime) is obtained by requiring $x, \tau$ to be real, so that $z$ is complex and $\bar{z}$ is the complex conjugate of $z$.] The Hamiltonian density becomes $T_{00} /(2 \pi)=\left[T_{z z}+T_{\overline{z z}}\right] /(2 \pi)$, and the momentum density is $T_{11} /(2 \pi)=\left[T_{z z}-T_{\overline{z z}}\right] /(2 \pi)$. The continuity equation obeyed by $T_{\mu \nu}$ becomes $\bar{\partial} T_{z z}+\partial T_{\bar{z} z}=0$, where $\partial=\frac{1}{2}\left(\partial_{x}-\partial_{t}\right), \bar{\partial}=\frac{1}{2}\left(\partial_{x}+\partial_{t}\right)$; this corresponds to conservation of energy and momentum. In addition, invariance under rotations of space (in space dimension larger than one) and under Lorentz boosts requires that $T_{\mu \nu}$ be symmetric, which becomes simply $T_{z \bar{z}}=T_{\bar{z} z}$ in light-cone variables in two dimensions.

In the present case, the Hamiltonian density $T /(2 \pi)$ obeys

$$
\bar{\partial} T=0,
$$

and can be identified as $T=T_{z z}$, while $T_{\overline{z z}}=T_{z \bar{z}}=$ $T_{\bar{z} z}=0$. This has striking consequences. First, in addition to conservation of energy and linear momentum (the latter corresponds to angular momentum for the circular edge), we also have Lorentz/scale invariance. In the light cone coordinates, a Lorentz boost acts as $z \rightarrow e^{\theta} z$, $\bar{z} \rightarrow e^{-\theta} \bar{z}$, where $\theta$ is real, while for a scale transformation, $z$ and $\bar{z}$ are both scaled by the same factor. If we consider the transformation on $z$ only, then these transformations cannot be distinguished. The generator for either is the integral (divided by $2 \pi$ ) of the chiral dilatation current $j_{D z}=T z$, which is divergenceless, $\bar{\partial} j_{D z}=0$, so $\int d x j_{D z}$ is conserved. If we restore the periodic boundary, the closest analogue of $j_{D z}$ is $T R \sin (z / R)$, for the drop of radius $R$. The Lorentz boost produces a Lorentz contraction of the circumference $2 \pi R$ of the drop; there is a natural rest frame in which the circumference is maximal (but one must examine both $z$ and $\bar{z}$ to see this geometry). 
For a general discussion of the relation between scale and conformal invariance in a Lorentz-invariant field theory, see Ref. 10]. For our purposes a useful sufficient condition for both is the existence of a symmetric stress tensor with vanishing trace, $T_{\mu}^{\mu}=0$ (where the summation convention is in effect, and indices are raised using the canonical inverse metric preserved by Lorentz transformations), which in light-cone variables means $T_{z} \bar{z}=0$. (We say "existence" because the stress tensor is not uniquely defined, and one can consider alternatives that differ by certain terms but all give the same Hamiltonian [10]; we can use any one such that $\bar{\partial} T_{z z}=0$.)

Conformal transformations in two dimensions form an infinite-dimensional algebra of symmetries (it includes the Lorentz and scale transformations) [8, [9]. An infinitesimal conformal mapping is described by a vector field with components $v^{z}(z), \bar{v}^{z}(\bar{z})$, where $\bar{\partial} v^{z}=\partial \bar{v}^{z}=0$, so $v^{z}(z)\left[\bar{v}^{\bar{z}}(\bar{z})\right]$ can be viewed as a function of $z(\bar{z})$ only; the transformation is $z \rightarrow z+\varepsilon v^{z}(z)$ (and similarly for $\bar{z}$ ) for $\varepsilon$ small, and $v^{z}=1, z$ yield the preceding special cases. (Each vector field should be analytic in some open set in the complex $z$ plane.) Again, the existence of a stress tensor with vanishing trace is a sufficient condition for invariance [10]. For our case, a divergenceless current corresponding to the transformation by $v$ is $j_{v z}(z)=v^{z}(z) T_{z z}$, which again obeys $\bar{\partial} j_{v z}=0$. Indeed, perhaps it should have been obvious from the beginning that the purely chiral dynamics would lead in the thermodynamic limit to an infinite number of conserved quantities; we are presently studying only those that generate space-time transformations.

We now have an algebra of operators $Q_{v}=$ $\int d x j_{v z} /(2 \pi)$ acting in the Hilbert space of the edge theory that forms a representation of the algebra of infinitesimal conformal mappings. The relations in this algebra are determined by the commutation relations of $T$, for which the most general form is (see e.g. Ref. [11])

$$
\begin{aligned}
{\left[T(z), T\left(z^{\prime}\right)\right] /(2 \pi i)=} & \delta\left(z-z^{\prime}\right) \partial T\left(z^{\prime}\right)-2\left(\partial \delta\left(z-z^{\prime}\right)\right) T\left(z^{\prime}\right) \\
& +(c / 12) \partial^{3} \delta\left(z-z^{\prime}\right) .
\end{aligned}
$$

The first two terms follow from the facts that $T$ generates translations in $z$ and has scaling dimension (conformal weight) 2 . For two vector fields $v^{z}, w^{z}$, the commutator of $Q$ 's is

$$
\left[Q_{v}, Q_{w}\right]=-i Q_{[v, w]}+(i c / 24 \pi) \int d z v^{z} \partial^{3} w^{z},
$$

where $[v, w]^{z}=v^{z} \partial w^{z}-w^{z} \partial v^{z}$, the Lie bracket of $v$ and $w$, is another vector field. The terms other than that containing $c$ are the relations defining the Lie algebra of conformal mappings. The additional term containing only the scalar $c$ times the identity operator is allowed for the usual reason that a given symmetry algebra may be represented projectively in quantum mechanics; in the present case, this term is unique modulo gauge transformations of the quantum states, under which $c$ is gauge invariant, so the term cannot be gauged away unless $c=0$ [12]. Returning to the system with periodic boundary condition, we form the Fourier modes (at $t=0) L_{n}=\oint d x e^{-i n x / R} T R /(2 \pi)+\delta_{n, 0} c / 24$. The corresponding algebra of these modes is the Virasoro algebra, with relations [8, 9]:

$$
\left[L_{n}, L_{m}\right]=(n-m) L_{n+m}+\frac{c}{12} n\left(n^{2}-1\right) \delta_{n+m, 0} .
$$

The central charge $c$ is a constant within a given edge theory. Self-adjointness of $T$ implies that the modes satisfy $L_{n}^{\dagger}=L_{-n}$. In a unitary theory, $c$ must be positive; no unitary CFTs exist for $c \leq 0$, except the trivial theory at $c=0$, in which the only state is the vacuum, and the stress tensor is zero. Thus we have established that the effective theory of the edge is a local unitary chiral CFT (in particular, it is relativistic, i.e. Lorentz invariant) [9].

To complete the discussion of conformal invariance, we should show that there is a "vacuum" state $|0\rangle$ in the edge theory that is invariant under the Möbius group, the group generated by the Virasoro generators $L_{0}, L_{-1}$, $L_{+1}$; then correlation functions in this vacuum possess Möbius invariance properties [8, 9]. The vacuum is also annihilated by the Fourier modes $L_{n}$ for all the positive modes $n>0$, as these lower the energy (or momentum), which is not possible for the vacuum. It is natural to expect that the unique ground state of lowest $M$, the existence of which we assumed, is this vacuum (they correspond in numerous examples). It certainly has the lowest energy in this sector. Then our precise definition of $\oint d x T$ in the vacuum sector is $\omega \Delta M=$ $\oint d x T /(2 \pi)+c / 24=L_{0} / R$ in the low energy region. However, at present we do not have a general argument that the ground state is invariant under $L_{-1}$, though $L_{0}|0\rangle=0$ holds for the ground state by the preceding definition. We should note that, although we can obtain the exact zero-energy many-particle wavefunctions for our special Hamiltonian $H_{0}$, calculating the norms of these, or operator matrix elements between them, remains very difficult. There are also other "sectors" (subspaces) of states in which one or more quasiholes is placed at the center of the drop, and it seems in examples that all sectors that should occur in the expected CFT arise in this way [4]. The lowest angular momentum state in each sector must be annihilated by all $L_{n}$ with $n>0$. The distinguishing feature of the vacuum is that it is annihilated by $L_{0}$ and $L_{-1}$ also. The lowest angular momentum state in each sector differs in $M$ from $M_{0}$ by amounts of order $N$, and currently we do not have a way to extract the expected finite difference of order 1 in conformal weight (energy times $R$ ). It seems natural that the sectors with higher $M$ for the same $N$ values are excited to positive energies in the edge theory (suitably defined), but this has not been proved. If we could confirm this, it would confirm the identification of the ground state with the vacuum, because in a unitary CFT the conformal weights of all states other than the vacuum are positive.

The presence of other conserved quantities in the original LLL model leads to further conserved quantities in 
the effective theory of the edge. Thus, particle number is always conserved, and there is a corresponding divergenceless Noether current in the full theory of bulk and edge (though the expression for this LLL current is not entirely obvious). Then there is also a corresponding divergenceless current $j_{z}$ in the edge theory, which is now chiral (its counterpart is $j_{\bar{z}}=0$ ) and obeys $\bar{\partial} j_{z}=0$. This is present in all QH systems. To make the discussion somewhat more general, let us consider also the case in which the particles carry spin $1 / 2$, the special Hamiltonian is invariant under $\mathrm{SU}(2)$ acting internally on the spin, and the ground state is an $\mathrm{SU}(2)$ singlet. (This serves as a paradigm for larger Lie algebras acting as internal symmetries, also.) Then there are local self-adjoint chiral spin current operators $j_{a z}$, where $a=1,2,3$ labels a basis for the adjoint representation of $\mathrm{SU}(2)$. All such currents are divergenceless, for example $\bar{\partial} j_{a z}=0$, and consequently have scaling dimension (conformal weight) 1 [1], 13]. Because of conformal invariance, the most general allowed form [11] of the commutators (or the operator products) of the currents are those for an affine Lie (Kac-Moody) algebra for SU(2) [13]. Thus the currents are good conformal fields (no logarithms in their correlators). The existence of such currents in a unitary CFT implies that $c \geq 1$, ruling out the trivial case $c=0$ among others. The affine Lie algebra contains a single parameter $k$ which must be a positive integer (or $k=0$ in the trivial case), as a consequence of the unitarity of the theory. For the particle number or U(1) symmetry, the affine Lie algebra simplifies as the structure constants vanish, and the corresponding $k$ is positive but need not be an integer; instead, given a suitable normalization of the $\mathrm{U}(1)$ current $j_{z}$, it corresponds to the filling factor (Hall conductivity) $\nu$ of the QH system. In the spin case, $k$ is a quantized Hall conductivity for spin. Another way to state the affine Lie algebra symmetry, similar to what we did for conformal invariance, is that if $w^{a}(z)$ is a vector field transforming in the (co-)adjoint representation, with $\bar{\partial} w^{a}=0$, then $j_{w z}(z)=\sum_{a} w^{a}(z) j_{a z}(z)$ is a divergenceless vector field: $\bar{\partial} j_{w z}=0$.

\section{EXAMPLE}

Next we will consider an example that at one time was considered to be a counterexample to the notion (proposed in MR [1]) that the effective theory of the edge is a CFT and is the same one that appears in describing the ground state wavefunction as a correlator in a Euclidean CFT. This example is the Haldane-Rezayi (HR) state [3]. It is the spin-singlet zero-energy ground state of a special "hollow-core" LLL Hamiltonian for fermions with spin $1 / 2$. The ground state wavefunction is a Laughlin factor times a correlator in the non-unitary CFT of symplectic fermions [1]. Later, the complete space of zero-energy wavefunctions was obtained [4]. The edge excitations above the ground state were naturally understood in terms of the charge degree of freedom associated with the current $j_{z}$, and neutral fermions carrying spin $1 / 2$ under the internal $\mathrm{SU}(2)$, and with conformal weight one. The latter would be natural if the edge theory were the non-unitary symplectic fermion theory. In order to construct a unitary theory, a theory of spin $1 / 2$ fermions with local spin currents, but without Lorentz invariance was proposed [4]; the correlators of the spin currents contained logarithms and did not have the affine Lie algebra form. An alternative proposal [14] for the fermion sector of the theory was a unitary Dirac fermion CFT, which gives the same Hilbert space and energy spectrum up to a shift in the zero of energy, but has nonlocal $\mathrm{SU}(2)$ spin currents 15] (the conformal weight of the fermions is then $1 / 2$, because the vacuum is identified with a distinct sector from the ground state above; this construction is possible only because two sectors possess ground states annihilated by the respective $L_{-1}$ in each fermion theory, when zero modes are absent as they are here). The upshot of all these works seems to be that no fully local unitary theory for the edge of the HR state exists, and this is in part a consequence of the (conformal) spinstatistics theorem in $1+1$ dimensions for unitary Lorentz invariant fermion theories. The apparent conflict with the arguments of the present paper is resolved because of the argument in Ref. [16] that the bulk of the system is not fully gapped; the fermion sector is gapless. Then it is not clear that there is any gapless edge spectrum (described perhaps by the zero-energy eigenstates of $H_{0}$ ) distinct from the general gapless excitations of the bulk. Several other cases of non-unitary CFTs (as well as some with other pathologies) that arise in a similar way in $\mathrm{QH}$ systems are also argued to be gapless in the bulk [5, 16]. No examples are known to me in which a non-unitary CFT arises from the bulk wavefunction, and the bulk is known to be fully gapped (but note that in general deciding on the latter issue may not be possible in numerical work because of finite size restrictions, and it requires the construction of a theory for the bulk, which for many QH systems has not been done).

\section{FURTHER DISCUSSION}

Now we return to the general discussion. If we consider all the local operators in the edge theory, then it makes sense to classify them according to representations of the conformal (Virasoro) algebra. Like the states of the edge theory, they form highest-weight representations, which means that their conformal weight ( $L_{0}$ eigenvalue) cannot be lowered indefinitely. Moreover, by applying a local field to the vacuum state, one obtains another state. The two-point correlation function of pairs of local operators then becomes essentially the inner product of the corresponding states, and is finite (possibly zero). Hence the local operators correspond to states in a positive definite space, on which the Virasoro algebra acts [8, 9]. It follows that they must form unitary representations of the algebra, as the states do. In particular, the represen- 
tation generated by a highest weight (such an operator is called a conformal primary field) must be irreducible. This eliminates logarithmic effects from all correlators of such fields. Further, in a unitary CFT, the conformal weight of a primary field must be non-negative (it can be zero only for the identity operator).

In our present situation, all local operators $\mathcal{O}$ obey $\bar{\partial} \mathcal{O}=0$, and there are corresponding conserved quantities. These operators acting on the vacuum span the vacuum sector of states, and there is an operator corresponding to each state. These operators form an algebra called the chiral algebra of the QH edge system. The chiral algebra contains the Virasoro and U(1) particle number current algebras, the current algebra for spin and so forth (if any), and also further operators. (We should note that the remaining sectors of states other than the vacuum sector are not created from the vacuum by using local operators. Indeed, in the QH system, they must be created by acting with some quasihole operator located somewhere in the bulk. They can be viewed as corresponding to states in the non-vacuum sector, nonetheless.)

As examples of local primary operators other than $T$ and the currents, we can consider the particle creation and annihilation operators. These are local as the corresponding operators (which are projected to the LLL) are local in the full bulk theory, and can be applied close to the edge. If the underlying particles are bosons (a situation that may possibly be realizable in rotating cold trapped atoms), then these operators commute when well separated, and similarly for fermions (as in QH systems), they anticommute. This can be extracted from correlation functions (defined using time-ordered products, as usual), which must be single valued in both cases, which leads to (anti-)commutation of the operators, and hence using conformal invariance the conformal weight of the primary field must be an integer in the boson case. Indeed, this is true for all strictly local operators. (The single-valuedness follows by considering the function first in the full bulk theory, where it follows from the general definition of the particle field operators, say using imaginary time; it is not lost by passing to large separation and low energies, near the edge, to obtain the corresponding function in the edge theory. This argument holds for any effective theory of the edge, even without conformal invariance, not only for the special Hamiltonian used here. Hence the claim in Ref. [17] that the operators for electrons in a chiral effective edge theory might not anticommute at large separation and equal time is incorrect.) In the case when the underlying particles are fermions, the field operators in the edge theory obey anticommutation relations, and the correlation functions are single-valued, but the fermion field operators must have half-odd-integer conformal weight. (Sometimes fermion fields are viewed as not strictly local, because they do not commute at large spatial separations.) Then the natural boundary condition on these fields, which must be the same in all sectors, in the cylindrical spacetime we are considering, is antiperiodic [9]. In this situation the chiral algebra in fact becomes a chiral superalgebra (in the "Neveu-Schartz" sector), as some of the fields obey anticommutation relations. Examples include the Laughlin states for fermions at $\nu=1,1 / 3, \ldots$, and the MooreRead state at $\nu=1 / 2$ (or $5 / 2$ ). When the conformal weight of the particle field is $3 / 2$, it generates a superconformal algebra (which also includes $T$ and the current $j_{z}$ ) at least in several examples, including the Laughlin $1 / 3$ state, the Moore-Read 1/2 state, and all other members of the Read-Rezayi series with $M=1$ also [1, 4, [6].

\section{BEYOND SPECIAL HAMILTONIANS}

Now we turn briefly to the problems that occur beyond the realm of special Hamiltonians. First, for more general local bulk Hamiltonians that can be deformed to some special Hamiltonian without crossing a bulk phase transition, the (fully-gapped) topological phase in the bulk remains the same as for the special Hamiltonian. In this situation, the edge theory should remain chiral and local, but will in general be perturbed by addition of terms (integrals of a local operator) to the Hamiltonian as the bulk Hamiltonian is perturbed away from the special one. (Actually, changes in the Hamiltonian near the edge can cause edge reconstruction, in which case the edge does not remain chiral; we do not consider this here.) Then the Hamiltonian density of the edge theory may contain extra local operators added to the stress tensor $T$ of the CFT. Such perturbations may be classified as relevant, irrelevant, or marginal under the renormalization group. These occur when the scaling dimension (which is the same as the conformal weight in the present chiral case) is $>2,<2$, or $=2$, respectively. Irrelevant operators produce no essential change and can be dropped at sufficiently low energies. Relevant ones cause a flow to some other fixed point, which is still a chiral theory. We note that in a chiral theory, the perturbations generally break Lorentz invariance. The low-energy fixed point may still be conformal, perhaps with some redefinitions (for example, an interesting example is two chiral Majorana fermi fields $\psi_{1}, \psi_{2}$, perturbed by a coupling $i m \psi_{1} \psi_{2}$ where $m$ has dimension 1; the low-energy fixed point is conformal provided one shifts the zero of momentum for the fermions by constants proportional to $\pm m$ ).

Marginal perturbations can be classified as marginally relevant, marginally irrelevant, and exactly marginal. The first two have similar effects as the relevant and irrelevant cases, respectively. In the exactly marginal case, the coefficients of the perturbation are not renormalized at any order in perturbation theory. As examples, any chiral CFT has an exactly marginal perturbation of dimension 2 , namely that by the stress tensor $T$. This has the effect of changing the velocity of all the edge excitations away from $v$, thus violating the Lorentz invariance of the original theory, though of course it is replaced by another Lorentz invariance. If the CFT is a tensor prod- 
uct of theories, which means that the stress tensor is a sum of those in two decoupled theories, $T=T_{1}+T_{2}$ (the theories may still be coupled through superselection rules on the non-vacuum sectors or quasiparticle operators), then adding a multiple of say $T_{1}$ to the Hamiltonian $T$ changes the velocity in theory 1 but not in theory 2 . More generally, such a theory can be perturbed by an operator $\mathcal{O}_{1} \mathcal{O}_{2}$ with a factor $\mathcal{O}_{i}, i=1,2$ from each theory, with $\operatorname{dim} \mathcal{O}_{1}+\operatorname{dim} \mathcal{O}_{2}=2$ to ensure it is marginal. If it is exactly marginal, this can have an effect similar to changing the velocities of the two theories, but may also cause mixing of the modes. For example, with more than one chiral boson field $\phi_{1}, \phi_{2}, \ldots$, as in various abelian QH states, a term $\partial \phi_{1} \partial \phi_{2}$ can be added, and is exactly marginal (see e.g. Ref. [18]). Such problems must be treated case by case, and we cannot go further here. But the point is that for such a Hamiltonian in the bulk, the edge theory is not strictly conformal, but is a perturbation of a CFT.

If there is no known special Hamiltonian that produces a particular topological phase, such as in many cases including Abelian states believed to occur in the QH system, the edge theory may or not be chiral (it must still be local and unitary when the bulk Hamiltonian is). For chiral cases, the preceding discussion of perturbations of a CFT still applies. We have no general argument that the edge theory for a generic Hamiltonian in such a phase must be a perturbation of a chiral CFT, but we expect this to be the case. However, if one can tune parameters of the theory so that all low-energy edge excitations propagate with a common velocity, while remaining in the same bulk topological phase, then the arguments of this paper still go through. For non-chiral cases, the discussion of perturbations of a CFT is similar, though now operators perturbing a CFT may contain parts from both right- and left-moving sectors, but must still be local. Again, see Ref. [18] for exactly-marginal examples. In these cases, it is less clear if there is a way to establish the existence of an underlying CFT. Finally, in all cases one can also consider long-range perturbations of the bulk theory, such as the Coulomb interaction, which leads to a long-range current-current interaction in the edge theory, which spoils Lorentz invariance.

\section{SOME RECENTLY-PROPOSED TRIAL STATES}

Now we turn to an application of the above argument, that the effective field theory of the edge of a gapped topological phase must be both conformally invariant and unitary, to some trial states of recent interest. We will find that for infinitely many of these the number of edge excitations they would have is not compatible with any unitary theory.

The trial wavefunctions in question [19, 20, 21, 22] are generalizations of the paired and clustered states of spinpolarized particles, such as those in Refs. [1, ㅎ, , 7]. In the simplest cases, which always describe bosons, the functions are defined by requiring them to vanish as the $R$ th power of separation $(R=2,3, \ldots)$ as $k+1$ particles come to the same point $(k=1,2, \ldots)$. For small enough $R$ or $k$, this produces a unique ground state, and reasonable numbers of excited edge (and also quasihole) states [23]. For $k>1$ and $R>3$, further conditions are required, which may be described in terms of the relation with " $(k, R)$ admissible partitions"; for $R>2$ it is required that $k+1$ and $R-1$ be coprime. (Further states are easily obtained from any of these by multiplying by a power of the Laughlin-Jastrow factor.) These functions are all given by Jack polynomials in the coordinates $z_{i}$ 20, 24]. Then the $R=2$ cases are the states labeled by $k$ (and $M=0$ ) in Ref. [6], where $k=1$ is the Laughlin state [7], and $k=2$ is the Moore-Read state [1]. The case $k=2, R=3$ is the so-called Gaffnian state [19]. Apart from a power of the Laughlin-Jastrow factor, the latter is a conformal block (correlator) in the non-unitary CFT known as the $M(5,3)$ minimal model [19] (we follow the conventions of Ref. [9]). It is known [24] that these Jack polynomials are related in this way to minimal models for $k=2$ (and all $R$ ), and to minimal models for $W_{k^{-}}$ algebras (the $W_{2}$ algebra is simply Virasoro) for $R=2$ (and all $k$ ); it is conjectured [24] to hold for all $k$ and $R$. Including the charge sector, the fusion rules for the bulk are known to be those of $\mathrm{SU}(R)$ level $k$ [25].] This conjecture is supported by recent work [26]. All cases with $R>2$ are non-unitary CFTs. In the cases at $k=1$ or $R \leq 3$, there are (local) special Hamiltonians involving interactions among at most $k+1$ particles for which these states are (at least conjecturally) the unique zeroenergy ground states. Bernevig and Haldane [20] state that for $k>1$ and $R>3$ they did not find a local Hamiltonian with $n$-body interactions for any $n$ that produces the Jack trial wavefunctions and no others. Even when such a Hamiltonian exists, it is not established whether it is gapped or gapless in the thermodynamic limit. The authors of Ref. [19] acknowledge that their special Hamiltonian may be gapless in this limit.

We will consider for the case $k=2$ the "counting formulas" that give the dimension of the spaces of trial wavefunctions for edge excitations of a disk as discussed above. Earlier results of this type, and also some for zero-energy quasihole states on a sphere, were obtained for $R=2$ and all $k$ (with no use of CFT) in Refs. [4, 5, 6, 19, 27, 28], prior to Refs. [20, 21, 22] [also, the connection of the counting formulas with those for $(k, 2)$ partitions was pointed out in Ref. [28]]. Simon et al. [19] give a general formula for the number of zeroenergy states (for their special Hamiltonian) for $n$ flux quanta added to the ground state on the sphere [see eq. (5) in Ref. [19]]. Using techniques outlined in Ref. 28], this can be generalized to count states at each angular momentum $L_{z}$, then used to obtain the number of edge states (if such they be), by taking the limit in which first $n \rightarrow \infty$, and then $N \rightarrow \infty$. The results can be presented as a generating or partition function, which is a 
series in an indeterminate $q$, in which the coefficient of the term $q^{\Delta M}$ is the dimension of the subspace of zeroenergy states with angular momentum change $\Delta M$ from the ground state (as above). The result for the edge of the Gaffnian state is

$$
\frac{1}{(q)_{\infty}} \sum_{f=0}^{\infty} \frac{q^{f(f+1)}}{(q)_{2 f}}
$$

where $(q)_{m}=(1-q)\left(1-q^{2}\right) \cdots\left(1-q^{m}\right)$. We note that $1 /(q)_{\infty}$ is the partition function for the edge of the Laughlin state [4], and is always present in such formulas because of the ubiquity of the density excitations at the edge. The remaining factor can be identified as the character $\chi_{1,1}^{5,3}$ for the vacuum (or identity) sector of the $M(5,3)$ minimal model [see eq. (4.4) in Ref. [29]]. It seems to be a general result that the counting of edge states in the thermodynamic limit agrees with the vacuum sector of the CFT that underlies the trial wavefunctions.

We now recall more details about the minimal CFTs for the Virasoro algebra [8], which in general are denoted $W_{2}\left(p, p^{\prime}\right)=M\left(p, p^{\prime}\right)$, parametrized by a pair of coprime positive integers $p, p^{\prime}$ with $p>p^{\prime}$ [9]. For the minimal models, the primary fields $\phi_{r, s}$ are labeled by ordered pairs of integers $(r, s)$, with $1 \leq r \leq p^{\prime}-1,1 \leq s \leq p-1$ (the so-called minimal block in the Kac table). There is an equivalence $r \rightarrow p^{\prime}-r, s \rightarrow p-s$, and each inequivalent primary occurs just once in the theory. The conformal weights and central charge are given by [8]

$$
\begin{aligned}
c & =1-6 \frac{\left(p-p^{\prime}\right)^{2}}{p p^{\prime}}, \\
h_{r, s} & =\frac{\left(p r-p^{\prime} s\right)^{2}-\left(p-p^{\prime}\right)^{2}}{4 p p^{\prime}} .
\end{aligned}
$$

The unitary minimal models, which are the only unitary CFTs with $c<1$, are those in which $p^{\prime}=m, p=m+1$, with $m=2,3,4, \ldots$ (the case $m=2$ is trivial). In these $c=1-6 /[m(m+1)]$.

The effective central charge for a CFT, or for anything with similar counting formulas, can be defined in terms of the rate of growth of the number of states at high $\Delta M$ in the vacuum (or any other) sector. In a modularinvariant CFT, such as any of the $W_{k}$ minimal models, it can be obtained by using a modular transformation [9], and is given by $c_{\mathrm{eff}}=c-24 h_{\mathrm{min}}$, where $h_{\mathrm{min}}$ is the lowest conformal weight in the theory. In unitary theories, $h_{\min }$ is zero, so $c_{\text {eff }}=c$. For the Virasoro minimal models, $h_{\text {min }}$ is the lowest value of $h_{r, s}$ in the minimal block. Using Bezout's lemma, and the fact that $p$ and $p^{\prime}$ are coprime, one obtains $h_{\min }=\left[1-\left(p-p^{\prime}\right)^{2}\right] /\left(4 p p^{\prime}\right)$, which is negative in all non-unitary cases. That is,

$$
c_{\text {eff }}=c-24 h_{\min }=1-\frac{6}{p p^{\prime}} .
$$

For the counting of QH trial wavefunctions, we will always remove the contribution 1 to both $c$ and $c_{\text {eff }}$ that is due to the charge sector. Then for the Gaffnian case $M(5,3)$ the central charge is $c=-3 / 5$, while the effective central charge is $c_{\text {eff }}=3 / 5$. More generally, the series of Jack states with $k=2$ are known to be (apart from the charge sector) conformal blocks of the minimal model $M(R+2,3)$ [24], and the counting formulas for these lead to the effective central charge $R /(R+2)$ [30], as we discuss further below. [A numerical check was carried out in Ref. 22]; note that these authors call this quantity the "thermal Hall conductivity", on the assumption that the bulk of the system is gapped (for some Hamiltonian) [16].]

Now we come to the crucial point. Even if the trial wavefunctions are conformal blocks in a non-unitary CFT, they might describe a gapped bulk phase and its edge excitations. If there is a special Hamiltonian for which the trial functions are zero-energy eigenstates, and which has a gap in the bulk, then we have seen that the effective theory of the edge must be a unitary CFT, which must be different from that used in the trial wavefunctions if the latter is non-unitary. In this case, its $c_{\text {eff }}$ value, defined by counting the excitations, must equal the central charge $c$ of the unitary CFT. For the cases with $k=2$, this value is $c=R /(R+2)$. As this is less than one, the allowed values of $c$ for a unitary theory are very restricted, and one finds that most values of $R$ do not correspond to such a value. The only values of $R$ for which $c=R /(R+2)$ corresponds to a unitary representation of Virasoro are given by

$$
R+2=m(m+1) / 3
$$

with either $m$ or $m+1$ divisible by 3 (but not by 9 ). After the Moore-Read case $R=2$, the next such values are $R=8,12,42, \ldots$ That is, for $R \neq m(m+1) / 3-2$ there is no unitary CFT that is an acceptable candidate to be the edge theory of these trial functions. In particular, this argument applies to the Gaffnian $[M(5,3)$ minimal model], for which a special Hamiltonian does exist. We conclude that it cannot describe a gapped topological phase, a conclusion also discussed in Refs. [19, 31]. Most likely, the special Hamiltonian is gapless in the thermodynamic limit.

For the cases $R=m(m+1) / 3-2$ that are not excluded by the above argument, we can examine the counting of states in more detail, if we can show that the generating or partition function of the edge excitations is given by the character for the $M(R+2,3)$ minimal model. For the primary field labeled $r, s$, this character can be written in the form [9]

$$
\chi_{r, s}^{p, p^{\prime}}=\frac{1}{(q)_{\infty}} \sum_{\lambda=-\infty}^{\infty}\left(q^{\lambda^{2} p p^{\prime}+\lambda\left(p r-p^{\prime} s\right)}-q^{\left(\lambda p^{\prime}+r\right)(\lambda p+s)}\right)
$$

(we omit the factor $q^{h_{r, s}}$ that is usually included). In every case, it can also be written in a "fermionic" form 32], similar to but usually more complicated than that for the $M(5,3)$ example in eq. (6) above. Our interest is 
in $r=s=1$. For the edge excitations of the Jack states, or the $(2, R)$ admissible partitions, the information given in Ref. 22] for $k=2$ is insufficient to answer the question, and contains undefined notation. Fortunately, the full results and notation were given by Feigin and coworkers [30], and using their results one can show that as $N \rightarrow \infty$ (with $N$ even) the counting of the $(2, R)$ admissible partitions and of the corresponding polynomials exactly coincides with the fermionic form of the character $\chi_{1,1}^{R+2,3}(q)$ for all $R$, after removing the factor $1 /(q)_{\infty}$ for the charge sector (the value of $c_{\text {eff }}$ then follows). The remaining question at issue is whether these characters can be equal for distinct pairs $p, p^{\prime}$ of coprime integers $\left(p>p^{\prime}>0\right)$ such that $p p^{\prime}$ is the same (in our particular case, one of the pairs is unitary, $\left.p=m+1, p^{\prime}=m\right)$. However, one can see that given a series of the form (11), the values of $p, p^{\prime}$ are uniquely determined, which rules out distinct pairs. Hence for $k=2$ no unitary CFT exists for any value $R>2$.

For the trial functions (Jack polynomials) with $k=2$, $R>3$, there appear to be no local special Hamiltonians. The argument still shows that there can be no unitary CFT that accounts for the "edge" trial states when $R>2$. In the absence of a Hamiltonian that is local and produces these zero-energy states and no others, it is unclear if it is meaningful even to ask if these functions represent edge excitations of a topological phase. The argument would still apply for a Hamiltonian that is gapped in the bulk, but for which the trial states are not eigenstates, if it is claimed to be in the same phase as those trial states (and even when it has different velocities for the charge and other excitations on the edge). If there is some non-local Hamiltonian that has a bulk gap and these trial states as zero-energy edge states, then the edge effective field theory still cannot be conformally invariant, which would not be surprising in view of the non-locality. Such a non-local theory would presumably be physically unacceptable in any case.

A last possibility might be that the trial ground state for the disk corresponds to an excited state on the edge, and vice versa, as mentioned for the Haldane-Rezayi state earlier. This seems to be inconsistent in all cases, as the identity in the fusion rules for bulk excitations is fixed. The fusion rules [25] and the identification of the identity for the bulk and the edge must agree.

For the models with $k>2$, the argument of this section does not go through as the values of $c_{\text {eff }}$ are bigger than 1 (for $R>2$ ), where the constraint of unitarity on Virasoro representations is very weak. It would be natural to try to generalize it using analogous statements for $W_{k}$ minimal models, if we could show directly that the necessary local conserved higher spin currents (of weights $3,4, \ldots, k)$ exist in the quantum-mechanical edge theories of these states. The unitary $W_{k}$ minimal models have central charge of the form

$$
c=(k-1)\left(1-\frac{k(k+1)}{m(m+1)}\right),
$$

where $m=k+1, k+2, \ldots 33$. In the lowest case $m=$ $k+1$, the $W_{k}$ minimal model is the $\mathbf{Z}_{k}$ parafermion theory used in Ref. [6]. Then for the $R=2$ cases, which are the Read-Rezayi states [6], the unitary boundary theory does exist, and including the charge sector is $\mathrm{SU}(2)$ level $k$; the higher spin currents must exist in the low-energy effective theories of these edges, which lends some support to the idea.

Bernevig and Haldane conjecture 22] that the effective central charge values arising from the Jack polynomial states are given by $c_{\text {eff }}=(k-1) R /(R+k)$ in all cases. These agree with $c$ in a unitary $W_{k}$ minimal model only if

$$
R+k=\frac{m(m+1)}{k+1}
$$

Clearly, most values of $R=3,4, \ldots$ (with $R+k$ and $k+1$ coprime) do not satisfy this condition. However, at present we do not know that the $W_{k}$ higher-spin currents necessarily exist, even when a special Hamiltonian does. Hence we have not yet eliminated the possibility that some other unitary CFT exists that would agree with the values of $c_{\mathrm{eff}}$ in the cases $k>2, R>2$.

\section{CONCLUSION}

To summarize, a chiral edge theory of a local Hamiltonian that has a gapped spectrum in the bulk (in the thermodynamic limit) and a single velocity for the edge excitations must be both conformally invariant and unitary. This applies in particular for many known special Hamiltonians for which the edge excitations can be identified as zero-energy eigenstates whose angular momentum differs from that of the ground state by amounts of order 1 . Then for many examples of proposed trial wavefunctions, including the Gaffnian state for which a local special Hamiltonian is known to exist (but is not known to be gapped in the bulk in the thermodynamic limit), the possibility that they represent a topological phase is ruled out because the number of edge excitations is not compatible with a unitary conformal field theory. The only remaining possibility for an interpretation of these states, if some Hamiltonian exists for which they are either exact eigenstates, or are in the same phase, is that either some excited states approach zero energy in the thermodynamic limit, or the Hamiltonian is significantly non-local.

We are grateful to N. Cooper and S. Simon for helpful discussions and correspondence. This work was supported by NSF grant no. DMR-0706195. 
[1] G. Moore and N. Read, Nucl. Phys. B360, 362 (1991).

[2] F.D.M. Haldane, Phys. Rev. Lett. 51, 605 (1983).

[3] F.D.M. Haldane and E.H. Rezayi, Phys. Rev. Lett. 60, 956, 1886 (E) (1988).

[4] M. Milovanović and N. Read, Phys. Rev. B 53, 13559 (1996).

[5] N. Read and E. Rezayi, Phys. Rev. B 54, 16864 (1996).

[6] N. Read and E. Rezayi, Phys. Rev. B 59, 8084 (1999).

[7] R.B. Laughlin, Phys. Rev. Lett. 50, 1395 (1983).

[8] A. Belavin, A. Polyakov, and A. Zamolodchikov, Nucl. Phys. B 241, 333 (1984).

[9] P. Di Francesco, P. Mathieu, and D. Senechal, Conformal Field Theory (Springer, New York, 1997).

[10] J. Polchinski, Nucl. Phys. B 303, 226 (1989).

[11] I. Affleck, Phys. Rev. Lett. 55, 1355 (1985).

[12] I.M. Gelfand and D.B. Fuks, Funct. Anal. Appl. 2, 342 (1968).

[13] V.G. Knizhnik and A.B. Zamolodchikov, Nucl. Phys. B 247, 83 (1984).

[14] V. Gurarie, M. Flohr, and C. Nayak, Nucl. Phys. B 498, 513 (1997).

[15] S. Guruswamy and A.W.W. Ludwig, Nucl. Phys. B 519 661 (1998).

[16] N. Read and D. Green, Phys. Rev. B 61, 10267 (2000).

[17] U. Zuelicke, J.J. Palacios, and A.H. MacDonald, Phys. Rev. B 67, 045303 (2003).

[18] F.D.M. Haldane, Phys. Rev. Lett. 74, 2090 (1995).

[19] S.H. Simon, E.H. Rezayi, N.R. Cooper, I. Berdnikov,
Phys. Rev. B 75, 075317 (2007).

[20] B.A. Bernevig and F.D.M. Haldane, Phys. Rev. Lett. 100, 246802 (2008).

[21] B.A. Bernevig and F.D.M. Haldane, Phys. Rev. B 77, 184502 (2008).

[22] B.A. Bernevig and F.D.M. Haldane, Phys. Rev. Lett. 101, 246806 (2008).

[23] S.H. Simon, E.H. Rezayi, and N.R. Cooper, Phys. Rev. B 75, 195306 (2007).

[24] B. Feigin, M. Jimbo, T. Miwa, and E. Mukhin, Int. Math. Res. Not. 23, 1223 (2002); arXiv:math.QA/0112127.

[25] E. Ardonne, arXiv:0809.0389.

[26] B.A Bernevig, V. Gurarie, and S.H. Simon, arXiv:0903.0635

[27] E. Ardonne, R. Kedem, and M. Stone, J. Phys. A 38, 617 (2005).

[28] N. Read, Phys. Rev. B 73, 245334 (2006).

[29] R. Kedem, T.R. Klasser, B.M. McCoy, and E. Melzer, Phys. Lett. B 307, 68 (1993).

[30] B. Feigin, M. Jimbo, and T. Miwa, arXiv:math.QA/0012193.

[31] N. Read, Phys. Rev. B 79, 045308 (2009).

[32] T. A. Welsh, Mem. Am. Math. Soc. 175N827 1 (2005); arXiv:math/0212154

[33] V.A Fateev and S.L. Lukyanov, Int. J. Mod. Phys. A 3, 507 (1988). 